Revista Internacional de Ciencias Podológicas

ISSN: 1989-5151

http://dx.doi.org/10.5209/RICP.57988

\title{
Fractura de Jones, ¿Tratamiento quirúrgico o tratamiento conservador?
}

Laura Posada López ${ }^{*}$

Resumen. La fractura de Jones se define como una fractura transversal localizada en la unión de la diáfisis con la metáfisis proximal en el quinto metatarsiano y es una de las lesiones más comunes que aparecen en el pie. Es una zona pobremente vascularizada, lo que conlleva altas tasas de no unión de la fractura. Se diagnostica con un exploración clínica, asociada a pruebas radiológicas, siendo necesarias tres proyecciones: lateral, antero-posterior y una oblicua de $45^{\circ}$. Hay distintas clasificaciones como son la de Torg et al. y Dameron et al., que nos ayudan a establecer un tratamiento, en función de la localización de la fractura y su evolución radiológica. El objetivo de este trabajo es realizar una revisión en la literatura científica para valorar las diferencias en los resultados entre el tratamiento quirúrgico y tratamiento conservador, en relación al tiempo de unión ósea y las posibles complicaciones, determinando así cual es el tratamiento óptimo para estas fracturas. Se llega a la conclusión de que el tratamiento conservador es una opción viable, pero conlleva mayores tasas de no unión y mayor tiempo de retorno a la actividad física que el tratamiento quirúrgico, aunque este puede llevar a una serie de complicaciones tales como la lesión del nervio sural o una posible fractura iatrogénica del metatarsiano. El tratamiento quirúrgico, que consiste mediante la fijación con un tornillo intramedular, tiene menor tiempo de reincorporación a la actividad habitual y mayores tasas de consolidación ósea por lo que será de primera elección en deportistas.

Palabras clave: fractura de jones; tratamiento quirúrgico; tratamiento conservador.

\section{[en] Jones Fracture. Surgical treatment or conservative treatment?}

\begin{abstract}
Jones' fracture is defined as a transverse fracture located at the junction of the diaphysis with the proximal metaphysis in the fifth metatarsal and is one of the most common lesions that appear in the foot. It's a poorly vascularized area, which leads to high rates of non-union of the fractures. It is diagnosed with a clinical examination, associated with radiological tests, three projections are necessary: lateral, antero-posterior and an oblique of $45^{\circ}$. There are different classifications such as Torg et al. and Dameron et al., which help us to establish a treatment, depending on the location of the fracture and its radiological evolution. The objective of this study is to perform a review in the scientific literature to evaluate the differences in the results between surgical treatment and conservative treatment, in relation to the time of union and the possible complications, thus determining which is the optimal treatment for these fractures. We conclude that conservative treatment is a viable option, but it leads to higher rates of non-union and greater time to return to physical activity than surgical treatment, although this may lead to a series of complications such as injury Sural nerve or a possible iatrogenic fracture of the metatarsal. The surgical treatment, which consists of the fixation with an intramedullary screw, has less time of reincorporation to the habitual activity and higher rates of consolidation by which it became the first choice in athletes.
\end{abstract}

Keywords: jones's fracture; surgical treatment; conservative treatment.

La autora declara no tener ningún tipo de interés económico o comercial.

Sumario: 1. Introducción. 1.1. Antecedentes y estado actual del tema. 1.2. Anatomía y Clasificación. 1.3. Mecanismo de Lesión. 1.4. Diagnóstico. 1.5. Objetivos del trabajo. 1.6 Material y método. 2. Desarrollo. 2.1. Tratamiento Conservador. 2.2. Tratamiento Quirúrgico. 2.2.1. Rehabilitación postoperatoria. 2.2.2. Contraindicaciones del tratamiento quirúrgico. 3. Discusión. 4. Conclusión. 5. Referencias bibliográficas.

1 Graduada en Podología. Facultad de Enfermería, Fisioterapia y Podología. Universidad Complutense de Madrid. lauraposada9520@gmail.com

* Dirección de correspondencia: Despacho 7. Podología General. Universidad Complutense de Madrid. Avenida Complutense s/n. 28040 Madrid. España. 
Cómo citar: Posada López, L. (2018) Fractura de Jones, ¿Tratamiento quirúrgico o tratamiento conservador?, en Revista internacional de ciencias podológicas 12(1), 35-44.

\section{Introducción}

\subsection{Antecedentes $y$ estado actual del tema}

La fractura de los huesos metatarsianos es una de las lesiones más comunes que se pueden encontrar en el pie, con una incidencia de 6,7 fracturas por cada 10.000 personas $^{1}$. El metatarsiano que con más frecuencia se fractura es el quinto metatarsiano (68\%), seguido de los metatarsianos centrales (10\%) y el primer metatarsiano $(1,5 \%)^{1}$

Robert Jones describió su propia patología en 1902, cuando se fracturó el quinto metatarsiano mientras bailaba ${ }^{2}$. Posteriormente fue definida como una fractura transversal en la unión metafisaria/diafisaria sin extensión distal más allá de la cuarta-quinta articulación intermetatarsal ${ }^{3,4}$.

Dameron et al. observaron que hay un predominio del sexo masculino en los pacientes más jóvenes, mientras que existe un predominio del sexo femenino en los pacientes con edad más avanzada ${ }^{5}$.

\subsection{Anatomía y Clasificación}

El quinto metatarsiano se articula proximalmente con el hueso cuboides y la base del cuarto metatarsiano ${ }^{6}$. Las fracturas de la parte proximal del quinto metatarsiano incluyen la zona de la tuberosidad, la zona de la metáfisis y de la diáfisis proximal ${ }^{6}$. Anatómicamente, la sección proximal del quinto metatarsiano es una región pobremente vascularizada, lo que conlleva a tener altas tasas de retraso en la consolidación ósea de la fractura $^{7,8}$. Smith et al. en 1992 investigaron el suministro de sangre del quinto metatarsiano, encontrando que surgía de tres fuentes posibles: la arteria nutriente, las perforantes metafisarias y las arterias del periostio ${ }^{9}$. Existe un área entre el suministro de la arteria nutriente y las perforantes metafisarias que corresponde a la zona de mala curación de la fractura de Jones y el riesgo de no unión en esta región ${ }^{9}$.

En cuanto a las inserciones de tejidos blandos que llegan a la parte proximal del quinto metatarsiano podemos destacar el tendón del peroneo brevis, el cual se inserta sobre la zona lateral de la tuberosidad, la banda lateral de la aponeurosis y el tendón del músculo tercer peroneo que se inserta dorsalmente en la zona de la metáfisis ${ }^{2,5}$.

A día de hoy hay bastante controversia sobre la clasificación y el tratamiento de las fracturas de Jones ${ }^{10}$. El tratamiento de las fracturas de la base del quinto metatarsiano puede variar dependiendo de la localización de la fractura y las expectativas del paciente ${ }^{11}$.

Otro factor que puede influir en el tratamiento es el tipo específico de fractura de Jones ${ }^{11}$.

Torg et al. propusieron la clasificación más comúnmente utilizada de las fracturas del quinto metatarsiano, en la cual describen la fractura de Jones como la fractura que implica la parte proximal de la diáfisis distal a la tuberosidad del quinto metatarsiano ${ }^{11}$. Su clasificación consistía en dividir la fractura de Jones según los hallazgos radiológicos y los tiempos de consolidación en tres tipos: Tipo I (aguda) es una fractura sin presencia de esclerosis intramedular ni antecedentes de traumatismo anterior, con una línea de fractura estrecha y mínima hipertrofia cortical; Tipo II (unión retardada) se caracterizan por tener una lesión previa y por presentar amplia línea de fractura y hueso nuevo perióstico y la fractura Tipo III (no unión) es una fractura con obliteración completa del canal medular por el hueso esclerótico, con líneas de fracturas anchas y antecedentes de trauma repetitivo y síntomas recurrentes ${ }^{11}$.

También hay otras clasificaciones para distinguir las fracturas del quinto metatarsiano con la fractura de Jones, como son la de Dameron et al. (1975) y Lawrence y Botte et al. (1993), que divide las fracturas en tres grupos anatómicos $^{12,13}$. La zona 1 se correspondería con fracturas por avulsión, la zona 2 con la unión de la metáfisis con la diáfisis (fractura de jones) y la zona 3 se localizaría en la diáfisis proximal $^{12,13}$.

La distinción entre la zona 2 (fractura de Jones) y la zona 3 (diafisarias proximales) se confunde comúnmente en la literatura, debido a su proximidad anatómica ${ }^{4,5,14}$.

En una revisión sistemática realizada por Dean et al. obtuvieron 19 estudios referidos 
con la clasificación de fractura de Jones ${ }^{15}$. Encontraron que la mayoría de los autores no diferenciaban entre fracturas que afectan a la cuarta/quinta articulación intermetatarsal de las fracturas más distales ${ }^{15}$. Llegaron a la conclusión de que generalmente se considera fractura de Jones a todas las fracturas del quinto metatarsiano localizadas aproximadamente a $1,5 \mathrm{~cm}$ distal de la tuberosidad del metatarsiano $^{15}$.

A diferencia de la zona 2 y la zona 3 , la zona 1 (fractura por avulsión) tiene un excelente potencial de curación, con lo cual las facturas que ocurren en esta zona pueden ser tratadas de manera conservadora, como ya veremos más adelante ${ }^{16}$. Esto se debe a que hay fuertes ligamentos entre la base del quinto metatarsiano y el cuboides, y la base del cuarto metatarsiano, que hacen que esta parte del quinto metatarsiano esté relativamente fija ${ }^{5,17}$. Por otro lado, la diáfisis del quinto metatarsiano es relativamente móvil ${ }^{4}$. Esta diferencia en la estabilidad hace también que las fracturas que ocurren en la unión metafisaria-diafisaria sean más susceptibles a la no unión de la misma ${ }^{4}$.

\subsection{Mecanismo de Lesión}

Una lesión del quinto metatarsiano conlleva una historia de trauma agudo o trauma repetitivo en la parte delantera del pie ${ }^{18}$.

Siguiendo la clasificación de Dameron et al., las fracturas de la zona 1 son resultado de lesiones por avulsión ${ }^{18}$. El mecanismo de estas fracturas es producido por un exceso de supinación del antepié junto con flexión plantar, dando lugar a la tracción de la banda lateral de la fascia plantar y del peroneo brevis ${ }^{18,19}$. Las fracturas de la zona 2 se producen como resultado de un episodio agudo ${ }^{12}$. El mecanismo exacto no se conoce, pero se cree que resulta de una gran fuerza de aducción en la parte delantera del pie, acompañada de mecanismos de flexión plantar del tobillo ${ }^{5,18}$. Las fracturas de la zona 3 generalmente son producidas por mecanismos de fatiga o estrés.

Son múltiples los factores que pueden contribuir al desarrollo de las fracturas por estrés incluyendo factores sistémicos, factores anatómicos y factores mecánicos ${ }^{20}$. El factor mecánico no está claro, pero se cree que es debido a que un músculo crea una fuerza localizada que supera la capacidad de tensión del hueso ${ }^{18}$.
Además, la alineación ósea del pie puede ser un factor predisponente para el desarrollo de lesiones en el quinto metatarsiano ${ }^{21,22}$. Por ejemplo, los pacientes con varo de retropié pueden tener un aumento de estrés en el quinto metatarsiano, aparte de ser más propensos a tener metatarso aducto, lo que provoca una sobrecarga de la columna lateral ${ }^{21,22}$.

\subsection{Diagnóstico}

La evaluación de un paciente con dolor en la base del quinto metatarsiano debe comenzar con una historia detallada que dé respuestas a las siguientes preguntas: ¿Hubo una lesión aguda? ¿Hubo una fractura previa? ¿Tuvo algún traumatismo previo?...,22.

Después de haber hecho una anamnesis exhaustiva, el clínico pasará a evaluar la sensibilidad a lo largo del quinto metatarsiano y los posibles signos de inflamación, como son la presencia de hinchazón, equimosis y dolor a la palpación ${ }^{1,22}$. El clínico debe realizar un diagnóstico diferencial con otras posibles lesiones, como pueden ser lesiones ligamentosas, lesiones del tendón peroneal $\mathrm{u}$ otro tipo de fractura ${ }^{1,22}$.

Entre las imágenes radiográficas indicadas ante una sospecha de fractura metatarsal, se encuentran tres proyecciones: lateral, anteroposterior, y una oblicua de $45^{\circ 23}$. Las fracturas agudas suelen requerir una radiografía posterior a la aparición inicial de los síntomas, en torno a los 10/14 días ${ }^{23}$.

En el caso de un traumatismo más complejo que involucre el mediopié, se recomienda una tomografía computarizada para descartar una posible luxación en la articulación de Lisfranc o para evaluar la curación de la fractura después de una operación, ya que nos permite identificar un posible defecto cortical en el aspecto plantar-lateral del quinto metatarsiano, aun cuando los hallazgos radiológicos se encuentran dentro de los parámetros de normalidad $^{23,24}$.

\subsection{Objetivos del trabajo}

Este trabajo tiene como objetivo realizar una revisión en la literatura científica para evaluar los tratamientos quirúrgico y conservador de la fractura de Jones, valorando las diferencias en los resultados entre ambos, en relación al tiempo de consolidación ósea y las posibles 
complicaciones, determinando así cual es el tratamiento óptimo para estas fracturas.

\subsection{Material y método}

La pregunta clínica que se utilizó para esta revisión sistemática se generó utilizando el formato PICO, planteando la siguiente pregunta: ¿Es mejor el tratamiento quirúrgico o el tratamiento conservador en pacientes con fractura de Jones? Esta pregunta se dividió en los términos de búsqueda "Jones fracture", "Jones fracture metatarsal" y "Jones fracture metatarsal treatment" y se buscó en cuatro bases de datos electrónicas (Pubmed, Web of Science, Scopus y Cohrane library) desde los últimos cinco años hasta marzo de 2017 (Tabla 1).

Fueron incluidos los artículos que informaron sobre las fracturas de Jones, así como otras fracturas metatarsianas. Los estudios que no compararon métodos operativos y no operativos, los que no intervinieron de forma aguda con el tratamiento quirúrgico o no se centraron en las fracturas de Jones fueron excluidos.

En la revisión no se incluyeron estudios de animales ni los artículos escritos en otro idioma que no fuera inglés. Solo fueron incluidos los artículos con alto grado de evidencia como son los meta-análisis, revisiones sistemáticas, revisiones y los ensayos clínicos controlados, siendo excluidos el resto de artículos (Tabla 2).

Al descartar los artículos repetidos entre las distintas bases de datos electrónicas, se obtuvieron un total de 1578 artículos, los cuales al aplicar los criterios de selección se quedaron en un total de 21 artículos. Tras analizarlos a texto completo se excluyeron 7 artículos porque no estaban relacionados con los términos de búsqueda y 1 artículo porque estaba en un idioma distinto al inglés. Finalmente se seleccionaron 13 artículos para el trabajo (Tabla 3).

\begin{tabular}{|c|c|c|c|c|}
\hline $\begin{array}{c}\text { Términos de } \\
\text { búsqueda }\end{array}$ & Pubmed & $\begin{array}{c}\text { Web of } \\
\text { Science }\end{array}$ & Scopus & $\begin{array}{c}\text { Cohrane } \\
\text { Library }\end{array}$ \\
\hline $\begin{array}{c}\text {-Jones fracture } \\
\text {-Jones fracture } \\
\text { metatarsal } \\
\text {-Jones fracture } \\
\text { metatarsal treatment }\end{array}$ & 1511 & 404 & 638 & 1 \\
\hline
\end{tabular}

Tabla 1: Resultados de la búsqueda bibliográfica sin incluir los criterios de selección 


\begin{tabular}{|l|l|}
\hline \multicolumn{1}{|c|}{ Criterios de inclusión } & \multicolumn{1}{|c|}{ Criterios de exclusión } \\
\hline Artículos relacionados con fractura de Jones ya & Artículos no publicados en inglés \\
sean: & Artículos con estudios de animales \\
-Meta-análisis & Artículos no relacionados con fractura de \\
-Revisiones Sistemáticas & Jones \\
-Ensayos clínicos controlados & Artículos que no cumplen con los criterios de \\
-Revisiones & inclusión: casos clínicos, caso control... \\
-Artículos de los últimos 5 años & \\
\hline
\end{tabular}

Tabla 2: Criterios de Selección

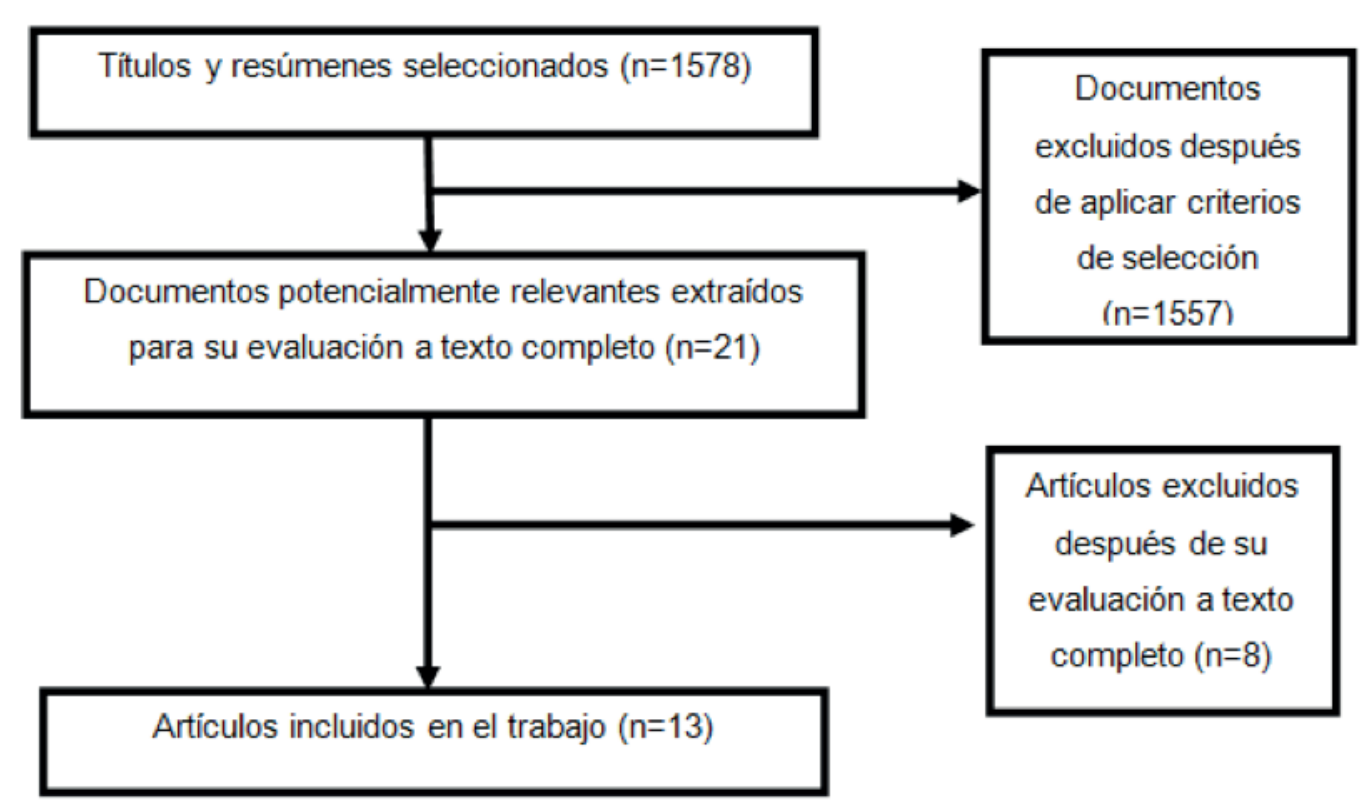

Tabla 3: Resultados de la estrategia de búsqueda 


\section{Desarrollo}

Las clasificaciones de Torg et al y la de Dameron et al se han convertido en la estrategia de tratamiento estándar para la fractura de Jones $4,11,12$. Se recomienda un régimen de tratamiento específico para cada tipo de fractura ${ }^{16}$.

\subsection{Tratamiento Conservador}

La elección del tratamiento no quirúrgico se debe basar en el paciente y el tipo de fractura individual $^{25}$. El tratamiento conservador se asocia, por lo general, con tiempos de curación ósea más largos, aunque evita los riesgos y las molestias de una cirugía ${ }^{20,26,27}$.

Como ya se ha mencionado anteriormente, según la clasificación de Dameron et al. y la de Lawrence y Botte et al., las fracturas por avulsión de la tuberosidad (zona 1), tienen un excelente potencial de curación ${ }^{12,13}$. Para las fracturas que ocurren la unión de la metáfisis con la diáfisis (fractura de Jones). Lawrence y Botte et al. sugieren inmovilización con una bota de escayola y evitar cargar el peso durante 6-8 semanas, a diferencia de las fracturas diafisarias de estrés (zona 3) que sugieren una inmovilización de hasta 20 semanas $^{13}$.

Torg et al. sugieren una estrategia de tratamiento de acuerdo a la clasificación que ellos describieron ${ }^{11}$. Las fracturas tipo I pueden ser tratadas de manera conservadora, mediante un yeso corto en la pierna durante un periodo de 3 a 12 semanas $^{11}$. Las fracturas tipo II pueden ser tratadas de manera conservadora o con intervención quirúrgica en función de la demanda funcional, ya que la mayoría de estas fracturas se curan con el tratamiento conservador en un periodo largo de tiempo, pero el tratamiento quirúrgico precoz puede reducir el tiempo de unión de la fractura y el tiempo de inmovilización ${ }^{11}$.

\subsection{Tratamiento Quirúrgico}

La intervención quirúrgica está indicada sobre todo a los atletas, con el objetivo de lograr la unión de la fractura en el menor tiempo posible, además de acelerar el retorno de la actividad deportiva, a la vez que reduce el riesgo de no unión y la recidiva de la fractura ${ }^{28}$. El tratamiento quirúrgico óptimo para la fractura de Jones aún no se ha determinado, pero el método ideal es uno que pueda soportar las fuerzas de torsión, así como las de tracción y las de flexión del metatarsiano ${ }^{29,30}$

Se ha demostrado que la técnica estándar utilizada en ensayos comparativos es la fijación mediante un tornillo intramedular canulado ${ }^{29}$. Este método ofrece gran resistencia a las fuerzas de tracción, pero poca compresión lateral de la corteza y poca resistencia contra las fuerzas de rotación ${ }^{29}$. Además, es un método que ofrece un rápido retorno de la actividad, permitiendo al paciente cargar el peso en un periodo relativamente corto de tiempo ${ }^{31}$.

Para mejorar la compresión lateral del hueso y proporcionar un mayor resistencia a las fuerzas de rotación, el cerclaje con alambre podría ser una alternativa viable, aunque esta opción puede causar mayor interrupción en el suministro sanguíneo del metatarsiano ${ }^{29,32}$. En algunos casos el cerclaje con alambre puede ser favorable si hay pequeños fragmentos en la fractura que no se puedan fijar con el tornillo intramedular ${ }^{33}$. Sarimo et al. (2006) trataron 27 pacientes con fractura de Jones con cerclaje, obteniendo muy buenos resultados ${ }^{34}$. Los pacientes comenzaron a soportar peso a las tres semanas y el tiempo medio de consolidación del hueso fue de 12,8 semanas $^{34,35}$.

El quinto metatarsiano tiene una curvatura lateral y su morfología es variable, por lo que es importante elegir el tipo de tornillo adecuado para cada paciente ${ }^{35}$. En la actualidad, existe una gran variedad de tornillos intramedulares que un cirujano puede seleccionar para la fijación de una fractura del quinto metatarsiano ${ }^{31,36}$. Existen los tornillos canulados y los sólidos ${ }^{31,36}$. La ventaja de un tornillo canulado es la gran precisión y la facilidad de la colocación del tornillo sobre una aguja guía ${ }^{31,36}$. Sin embargo, un estudio realizado por Glasgow et al (1996) informó del riesgo de recidiva en la fractura fijada con tornillos canulados ${ }^{37}$.

Un estudio reciente realizado por Scott et al (2015) determinó que un tornillo de 4,5 mm es el tornillo de diámetro más pequeño que se puede utilizar eficazmente para la fractura del quinto metatarsiano ${ }^{38}$. En cuanto a la longitud del tornillo DeSandis et al. comentan que rara vez el tornillo debe ser mayor de $50 \mathrm{~mm}$ y generalmente debe ser de $40 \mathrm{~mm}$ o menos para evitar la distracción de la fractura ${ }^{35}$. Además, se debe prestar especial atención a las personas mayores, ya que en esta población se deben evitar los tornillos excesivamente largos para reducir el riesgo de perforación de la corteza 
medial que puede conducir a la distracción de la fractura.

Un estudio realizado por Devries et al. (2011), comparó la fijación mediante tornillos canulados por acero inoxidable, con tornillo compuestos por titanio ${ }^{39}$. Llegaron a la conclusión de que ambos tenían tasas comparables de consolidación ósea, demostrando la ausencia de una ventaja clínica en la selección de uno u otro ${ }^{39}$.

De Lee et al (1983) describieron los pasos de la técnica quirúrgica para la fijación de la fractura de $\mathrm{Jones}^{20}$. A) Se realiza una incisión rectilínea para realizar una disección por planos y exponer la base del quinto metatarsiano entre los tendones peroneo lateral corto $\mathrm{y}$ peroneo lateral $\operatorname{largo}{ }^{20}$. B) Se inserta una aguja Kirchner en el canal medular del metatarsiano, que nos servirá de aguja guía para la inserción del tornillo, y se comprueba si está bien posicionado mediante fluoroscopio $\mathrm{u}$ otras técnicas ${ }^{20}$. C) Con un lápiz dermográfico estéril se marca en la piel la dirección de la aguja, para servir de guía para la inserción del taladro (si la marca no está pintada correctamente, el taladro puede penetrar el metatarsiano) ${ }^{20}$. D) Una vez insertado el taladro, se comprobará mediante el fluoroscopio su dirección y se insertará un tornillo intramedular canulado. Posteriormente se realizará una sutura por planos ${ }^{20}$.

\subsubsection{Rehabilitación postoperatoria}

La rehabilitación postoperatoria consiste, por lo general, en inmovilización con una férula de yeso durante una o dos semanas y posteriormente, el remplazo por una bota tipo Walker para caminar ${ }^{20,40,41}$. Esta bota reduce significativamente la presión en la base del quinto metatarsiano en la ambulación, en comparación con un zapato postoperatorio ${ }^{42}$.

A las seis/ocho semanas, el paciente podrá apoyar por completo el pie y podrá reanudar su actividad normal ${ }^{20,40,41}$.

Los pacientes atletas que se someten a cirugía deberían volver a su actividad deportiva solo si existe evidencia radiológica de la unión de la fractura, y el paciente presenta una clínica asintomática ${ }^{43}$. Podrán llevar una ortesis funcional mientras estén haciendo la actividad deportiva ${ }^{18,22}$.

\subsubsection{Contraindicaciones del tratamiento quirúrgico}

No hay evidencia de contraindicaciones específicas del tratamiento quirúrgico de la fractura de Jones ${ }^{44}$. Generalmente este tratamiento no está recomendado en pacientes con afectación vascular, neuropatía e infección local $^{44}$. La Diabetes mellitus no es una contraindicación absoluta, siempre y cuando el suministro vascular no este afectado ${ }^{44}$.

\section{Discusión}

Tras revisar la literatura para evaluar los diferentes resultados obtenidos mediante una terapia conservadora o quirúrgica, se han encontrado estudios que muestran diferencias significativas entre ambas opciones de tratamiento, como el de Yates et al. (2015) que realizó un meta análisis en el que muestra diferencias significativas entre el tratamiento quirúrgico en comparación con el conservador $(p<0.001)$, encontrando menores tasas de no unión en la primera opción, con un rango de 0 a $11 \%$, siendo mayor la tasa de no unión en la opción no quirúrgica con un rango de 11 a $50 \%{ }^{45}$.

Otras revisiones han visto resultados muy similares, expresando que los resultados obtenidos en las intervenciones quirúrgicas conllevaban menores tasas de no unión que el tratamiento conservador, además de menor tiempo de consolidación de fractura y reducción de las complicaciones ${ }^{10,14,15,46,47}$.

En cuanto a las complicaciones en el tratamiento, hubo más en los pacientes tratados de forma conservadora, que posteriormente requirieron manejo quirúrgico ${ }^{45}$. Un total de 38 de 132 pacientes (31\%) necesitaron cirugía después del tratamiento conservador en comparación con 9 de $106(8,5 \%)$ que necesitaron realizarse una intervención de nuevo ${ }^{45}$. Las complicaciones más comunes en ambos tratamientos eran: recidiva de la fractura, retraso de la consolidación ósea y la no unión de la fractura, además de que en el tratamiento quirúrgico muchos pacientes se quejaron de la incomodidad del tornillo ${ }^{45}$. Otras posibles complicaciones específicas de la fijación quirúrgica son la infección de la herida, la rotura parcial del tendón peroneo brevis, la irritación del nervio peroneal, la lesión del nervio sural, metatarsalgia y 
una posible fractura iatrogénica del hueso metatarsiano ${ }^{20,48}$.

Todos los estudios se componían principalmente de pacientes atléticos jóvenes, con un promedio de edad de 20,3 años según el estudio de Kavanaugh et al. (1978) en el que contaban sobre todo con atletas de nivel universitario; 25,6 años según Mologne et al. (2005), teniendo 36 de 37 pacientes militares de servicio activo; 27 años según Chuckpaiwong et al. (2008), la mayoría atletas de élite y 23 años por Ekstrand et at. (2013), también la mayoría deportistas de élite $19,26,32,41,45$. Además tanto Kavanaugh et al. como Chuckpaiwong et al. no encontraron diferencias estadísticamente significativas entre el índice de masa corporal o en la etnia que pudiesen tener los pacientes en relación con los términos de curación ${ }^{19,26}$.

\section{Conclusión}

Se debe distinguir que tipo de fractura presenta el paciente para poder establecer un buen tratamiento. Según la literatura científica, el tratamiento quirúrgico (mediante la fijación de un tornillo intramedular) es la mejor opción sobre todo en aquellos pacientes que necesitan una rápida reincorporación a su actividad habitual, como es el caso de los deportistas de alto nivel, ya que se ha encontrado menores tasas de no unión, menores complicaciones y menor tiempo de consolidación ósea de la fractura, recomendando el tratamiento conservador a los pacientes que presenta una fractura por avulsión o que no presenta esclerosis intramedular en pruebas radiológicas.

\section{Referencias bibliográficas}

1. Petrisor BA, Ekrol I, Court-Brown C. The epidemiology of metatarsal fractures. Foot ankle Int [Internet]. 2006 Mar ;27(3):172-4.

2. Jones R. I. Fracture of the Base of the Fifth Metatarsal Bone by Indirect Violence. Ann Surg [Internet]. 1902 Jun ;35(6):697-700.2.

3. STEWART IM. Jones's fracture: fracture of base of fifth metatarsal. Clin Orthop [Internet]. 1960; 16:190-8.

4. Zwitser EW, Breederveld RS. Fractures of the fifth metatarsal; diagnosis and treatment. Injury [Internet]. 2010 Jun ;41(6):555-62.

5. Dameron. Fractures of the Proximal Fifth Metatarsal: Selecting the Best Treatment Option. J Am Acad Orthop Surg [Internet]. 1995 Mar ;3(2):110-4.

6. Cheung CN, Lui TH. Proximal Fifth Metatarsal Fractures: Anatomy, Classification, Treatment and Complications. Arch trauma Res [Internet]. 2016 Dec ;5(4):e33298.

7. Ding BC, Weatherall JM, Mroczek KJ, Sheskier SC. Fractures of the proximal fifth metatarsal: keeping up with the Joneses. Bull NYU Hosp Jt Dis [Internet]. $2012 ; 70(1): 49-55$.

8. McKeon KE, Johnson JE, McCormick JJ, Klein SE. The intraosseous and extraosseous vascular supply of the fifth metatarsal: implications for fifth metatarsal osteotomy. Foot ankle Int [Internet]. 2013 Jan; 34(1):117-23.

9. Smith JW, Arnoczky SP, Hersh A. The intraosseous blood supply of the fifth metatarsal: implications for proximal fracture healing. Foot Ankle [Internet]. ;13(3):143-52.

10. Kerkhoffs GM, Versteegh VE, Sierevelt IN, Kloen P, van Dijk CN. Treatment of proximal metatarsal V fractures in athletes and non-athletes. Br J Sports Med [Internet]. 2012 Jul ;46(9):644-8.

11. Torg JS, Balduini FC, Zelko RR, Pavlov H, Peff TC, Das M. Fractures of the base of the fifth metatarsal distal to the tuberosity. Classification and guidelines for non-surgical and surgical management. J Bone Joint Surg Am [Internet]. 1984 Feb ;66(2):209-14.

12. Dameron TB. Fractures and anatomical variations of the proximal portion of the fifth metatarsal. J Bone Joint Surg Am [Internet]. 1975 Sep ;57(6):788-92.

13. Lawrence SJ, Botte MJ. Jones' fractures and related fractures of the proximal fifth metatarsal. Foot Ankle [Internet]. ;14(6):358-65.

14. Roche AJ, Calder JDF. Treatment and return to sport following a Jones fracture of the fifth metatarsal: a systematic review. Knee Surg Sports Traumatol Arthrosc [Internet]. 2013 Jun 6 ;21(6):1307-15.

15. Dean BJF, Kothari A, Uppal H, Kankate R. The Jones Fracture Classification, Management, Outcome, and Complications: A Systematic Review. Foot Ankle Spec [Internet]. 2012 Aug ;5(4):256-9.

16. Polzer H, Polzer S, Mutschler W, Prall WC. Acute fractures to the proximal fifth metatarsal bone: Development of classification and treatment recommendations based on the current evidence. Injury [Internet]. 2012 Oct ;43(10):1626-32. 
17. Lee KT, Park YU, Young KW, Kim JS, Kim JB. Surgical results of 5th metatarsal stress fracture using modified tension band wiring. Knee Surgery, Sport Traumatol Arthrosc [Internet]. 2011 May; 19(5):853-7.

18. Fetzer GB, Wright RW. Metatarsal Shaft Fractures and Fractures of the Proximal Fifth Metatarsal. Clin Sports Med [Internet]. 2006 Jan ;25(1):139-50.

19. Chuckpaiwong B, Queen RM, Easley ME, Nunley JA. Distinguishing Jones and proximal diaphyseal fractures of the fifth metatarsal. Clin Orthop Relat Res [Internet]. 2008 Aug ;466(8):1966-70.

20. DeLee JC, Evans JP, Julian J. Stress fracture of the fifth metatarsal. Am J Sports Med [Internet]. 1983 Sep ;11(5):349-53.

21. Yoho RM, Carrington S, Dix B, Vardaxis V. The Association of Metatarsus Adductus to the Proximal Fifth Metatarsal Jones Fracture. J Foot Ankle Surg [Internet]. 2012 Nov ;51(6):739-42.

22. Raikin SM, Slenker N, Ratigan B. The Association of a Varus Hindfoot and Fracture of the Fifth Metatarsal Metaphyseal-Diaphyseal Junction. Am J Sports Med [Internet]. 2008 Jul ;36(7):1367-72.

23. Rammelt S, Heineck J, Zwipp H. Metatarsal fractures. Injury [Internet]. 2004 Sep ;35(2):77-86.

24. Le M, Anderson R. Zone II and III fifth metatarsal fractures in athletes. Curr Rev Musculoskelet Med [Internet]. 2017 Mar ;10(1):86-93. Available from: http://ink.springer.com/10.1007/s12178-017-9388-5

25. Bowes J, Buckley R. Fifth metatarsal fractures and current treatment. World J Orthop [Internet]. 2016 Dec ;7(12):793.

26. Kavanaugh JH, Brower TD, Mann R V. The Jones fracture revisited. J Bone Joint Surg Am [Internet]. 1978 Sep ;60(6):776-82.

27. Granata JD, Berlet GC, Philbin TM, Jones G, Kaeding CC, Peterson KS. Failed Surgical Management of Acute Proximal Fifth Metatarsal (Jones) Fractures: A Retrospective Case Series and Literature Review. Foot Ankle Spec [Internet]. 2015 Dec ;8(6):454-9.

28. Hunt KJ, Anderson RB. Treatment of Jones fracture nonunions and refractures in the elite athlete: outcomes of intramedullary screw fixation with bone grafting. Am J Sports Med [Internet]. 2011 Sep; 39(9):1948-54.

29. Vertullo CJ, Glisson RR, Nunley JA. Torsional strains in the proximal fifth metatarsal: implications for Jones and stress fracture management. Foot ankle Int [Internet]. 2004 Sep ;25(9):650-6.

30. Lee SK, Park JS, Choy WS. LCP distal ulna hook plate as alternative fixation for fifth metatarsal base fracture. Eur J Orthop Surg Traumatol [Internet]. 2013 Aug ;23(6):705-13.

31. Portland G, Kelikian A, Kodros S. Acute surgical management of Jones' fractures. Foot ankle Int [Internet]. 2003 Nov ;24(11):829-33.

32. Ekstrand J, van Dijk CN. Fifth metatarsal fractures among male professional footballers: a potential career-ending disease. Br J Sports Med [Internet]. 2013 Aug ;47(12):754-8.

33. Wiener BD, Linder JF, Giattini JF. Treatment of fractures of the fifth metatarsal: a prospective study. Foot ankle Int [Internet]. 1997 May ;18(5):267-9.

34. Sarimo J, Rantanen J, Orava S, Alanen J. Tension-band wiring for fractures of the fifth metatarsal located in the junction of the proximal metaphysis and diaphysis. Am J Sports Med [Internet]. 2006 Mar ;34(3):476-80.

35. DeSandis B, Murphy C, Rosenbaum A, Levitsky M, O’Malley Q, Konin G, et al. Multiplanar CT Analysis of Fifth Metatarsal Morphology. Foot Ankle Int [Internet]. 2016 May ;37(5):528-36.

36. Pietropaoli MP, Wnorowski DC, Werner FW, Fortino MD. Intramedullary screw fixation of Jones fractures: a biomechanical study. Foot ankle Int [Internet]. 1999 Sep ;20(9):560-3.

37. Glasgow MT, Naranja RJ, Glasgow SG, Torg JS. Analysis of Failed Surgical Management of Fractures of the Base of the Fifth Metatarsal Distal to the Tuberosity: The Jones Fracture. Foot Ankle Int [Internet]. 1996 Aug ; 17(8):449-57.

38. Scott RT, Hyer CF, DeMill SL. Screw Fixation Diameter for Fifth Metatarsal Jones Fracture: A Cadaveric Study. J Foot Ankle Surg [Internet]. 2015 Mar ;54(2):227-9.

39. DeVries JG, Cuttica DJ, Hyer CF. Cannulated screw fixation of Jones fifth metatarsal fractures: a comparison of titanium and stainless steel screw fixation. J Foot Ankle Surg [Internet]. 2011 Mar; 50(2):207-12.

40. Mahajan V, Chung HW, Suh JS. Fractures of the Proximal Fifth Metatarsal: Percutaneous Bicortical Fixation. Clin Orthop Surg [Internet]. $2011 ; 3(2): 140$.

41. Mologne TS, Lundeen JM, Clapper MF, O'Brien TJ. Early screw fixation versus casting in the treatment of acute Jones fractures. Am J Sports Med [Internet]. 2005 Jul ;33(7):970-5.

42. Hunt KJ, Goeb Y, Esparza R, Malone M, Shultz R, Matheson G. Site-Specific Loading at the Fifth Metatarsal Base in Rehabilitative Devices: Implications for Jones Fracture Treatment. PM\&R [Internet]. 2014 Nov ;6(11):1022-9.

43. Larson CM, Almekinders LC, Taft TN, Garrett WE. Intramedullary screw fixation of Jones fractures. Analysis of failure. Am J Sports Med [Internet]. ;30(1):55-60. 
44. Yue JJ, Marcus RE. The Role of Internal Fixation in the Treatment of Jones Fractures in Diabetics. Foot Ankle Int [Internet]. 1996 Sep ;17(9):559-62.

45. Yates J, Feeley I, Sasikumar S, Rattan G, Hannigan A, Sheehan E. Jones fracture of the fifth metatarsal: Is operative intervention justified? A systematic review of the literature and meta-analysis of results. Foot (Edinb) [Internet]. 2015 Dec ;25(4):251-7.

46. Smith TO, Clark A, Hing CB. Interventions for treating proximal fifth metatarsal fractures in adults: a meta-analysis of the current evidence-base. Foot Ankle Surg [Internet]. 2011 Dec ;17(4):300-7.

47. Thevendran G, Deol RS, Calder JDF. Fifth metatarsal fractures in the athlete: evidence for management. Foot Ankle Clin [Internet]. 2013 Jun ;18(2):237-54.

48. Donley BG, McCollum MJ, Murphy GA, Richardson EG. Risk of Sural Nerve Injury with Intramedullary Screw Fixation of Fifth Metatarsal Fractures: A Cadaver Study. Foot Ankle Int [Internet]. 1999 Mar ;20(3):182-4.

49. Golanó P, Vega J, de Leeuw PAJ, Malagelada F, Manzanares MC, Götzens V, et al. Anatomy of the ankle ligaments: a pictorial essay. Knee Surg Sports Traumatol Arthrosc [Internet]. 2010 May ;18(5):557-69. 